\title{
"This is me with Augustine, February 21, 1943": Importance of Photographs in Everything Is Illuminated
}

\author{
Md Abu Shahid Abdullah, University of Asia Pacific
}

\begin{abstract}
Multimodal communication can simply be defined as communication through modes such as pictures, gestures, sign languages, and such. Photographs are relevant to literary studies because they too can narrate. Photographs can perform as unreliable narrators, drawing fuzzy borders between reality and imagination and destabilizing the concept of absolute truth. Photographs are embedded within collective memory and are able to build a foundation for remembrance. They demonstrate that memory in photos exceeds that of orality. In Jonathan Safran Foer's Everything Is Illuminated, photographs take a vital role along with written language. The paper aims at demonstrating the importance of photographs as a means to shed lights on constructs such as truth, emotions, memory and space. It deals with how photos communicate with people, how they can be differently perceived and why one should not accept their images as free from manipulation. The next part investigates what emotions photographs can stand for, and the connection between them and the be holder. Finally, the paper dwells on discourses photographs become intertwined with throughout the narration of the novel.
\end{abstract}

Keywords: communication; historiography; multimodal; photographs; reality; trauma

\section{Introduction}

A photograph of his grandfather and a woman named Augustine was the motivating factor behind and clue for Jonathan Safran's search for his family history, and the woman who saved his grandfather's life during the WWII. The initiation of his search for the past with the help of his Ukrainian translator Alex and his grandfather through the Ukraine results in finding Lista (the sister of Augustine). Through the testimony of Lista, Jonathan comes to know his family's past, and Alex shockingly discovers his grandfather's involvement in the death of his Jewish friend. This essay will discuss Jonathan Safran Foer's Everything Is Illuminated according to the importance of photographs, based on their ability to shed light on truth, memory, emotions and space. The aim is to unveil the tensions and subjectivity in the particular areas. Photographs are relevant to literary studies because they too can narrate. They possess more than a purely representational function of reality, just showing us our joys and pains of the past. The paper will commence with theories of photograph, concerned with how photos communicate with people, how they can be differently perceived and that one should not accept their images as free from manipulation. The next part will investigate what emotions photographs can stand for, and the connection between them and the beholder. Finally, the paper will dwell on discourses photographs become intertwined with throughout the narration of the novel. It will exemplify all the discussed points with examples from the novel.

\section{Theory of photographs}

\subsection{Watzlawick's Communication Model}

According to Paul Watzlawick's (1967) first axiom "Man kannnichtnichtkommunizieren", communication is never one-sided; the speaker is sending a message to the receiver(s), meaning there are always at least two parties involved simultaneously in the process, consciously and/or unconsciously (Rechtien, 2014). The two types are called verbal and non-verbal communication or dialogue and analogue communication in Watzlawick's words, with the latter being responsible for emitting signals via body language, facial expressions, which can be interpreted differently from individual to individual, from one culture to another (Rechtien, 2014). These signals can reveal more insight into what the other is really thinking; so, speech should not always be seen as the dominant medium of interaction. Photographs belong to non-verbal communication, which results in their complete interpretation not happening on the basis of so-called anthropological constants since the human perception is not universal but individualistic and culture-bound. Watzlawick's model of communication can be related to the photo of Augustine. The front 
side represents the analogue mode of communication, being the silenced moment of Safran's Grandfather, his alleged savior Augustine and her alleged parents. However, without an entity present from the photograph's process, to contextualize and denote its meaning this photo stands for authorial reticence. Nobody can make a clear statement of the credibility of whether the photo's true face is actually of Safran's grandfather and Augustine: "And how do you know that her name is Augustine?" (Foer, 2003: 60). The photo answers the reticence with a dialogue mode of communication. The words "This is me with Augustine, February 21 (1943: 60) are written on the back, which delivers a more factual and less-ambiguous message for the posterity. Although, the text-image relation may have been manipulated with and the text does not complement or complete the image, it leads to the estrangement of the photo in its entirety, similar to how the alleged parents become peripheral: "Her parents appeared very Russian, but she did not. She appeared American". Text can be disregarded as being embedded within an unreliable narration: a narration known to be misleading and one which fictionalizes events. Who can slip faithfully into the Grandfather's role of the past, bearing such minimal knowledge of his personality? Who can re-enact the state of his trauma in connection with the murdering of his wife and baby by the Nazis? Along with his unknown mental health, who can tell whether Jonathan's grandmother is trustworthy due to her brief information about the photo's history?:

"My grandmother gave it to my mother two years ago, and she said that this was the family that saved my grandfather from the Nazis. [...] No. We couldn't ask her anything about it. [...] She held on to the photograph for fifty years. If she had wanted to tell us anything about it, she would have" (Foer, 2003: 61).

Any secrets regarding the photo will accompany her to the grave. She is the closest source, still known to be alive and reachable.

\subsection{Top-down and Bottom-up Processes}

The mechanism of perceiving photographs can either occur in top-down or bottom-up processes (Weseley \& McEntarffer, 2010: 108-109). Top-down process refers to using one's empirical and background knowledge of the world and bottom-up refers to using only the individual features of the object, without incorporating one's past experiences, to construct a subjective meaning. Situated in another cultural circle, top-down processing could have the effect of jumping to conclusions because of varying cultural codes, forcing the perception to make use of stereotypical thinking and filling in the gaps with acquainted patterns. Augustine's photo is analyzed according to the top-down process. The three men estimate that she is most likely still alive, taking into consideration that she was probably around 15 to 18 years old in the picture and that she had family (mother and father) back then to support her. That the "family" was able to rescue somebody else signalizes that there is a great possibility that they were capable of saving themselves. The fact that Alex and Jonathan develop feelings for Augustine makes it naturally more difficult to give up hope.

Bottom-up process surfaces when Jonathan examines a photo from "In Case", which turns out to be of Alex's grandfather. He carefully studies it, turning his attention away, and then redirecting his attention once again, every time processing the different stimuli. To avoid being tricked by an optical illusion, he seeks confirmation of his growing belief in the candlelight. Then he positions the photo close to Alex, comparing his face with one, which turns out to be of his father as a young boy. Then the grandfather takes his turn, using candlelight and thoroughly studying the people from top till bottom. In the end, grandfather verifies and reveals his true identity during the war which leads him tell the story of his involvement in his Jewish friend Herschel's murder by the Nazis. Summed up, bottom-up can be more accurate in detecting "truths". The grandfather always said he is from Odessa, so top-down process would overlook individual features of him being on such a photograph, seeing as Alex or Jonathan's expectations had them thinking, he never set foot in that part of the country.

\subsection{Mimesis and Poiesis}

Photographs juggle between mimesis and poiesis. On the one hand, they depict reality or represent 
a fraction of it, but on the other hand, the person behind the lens selectively and unconsciously chooses and creates a manipulated setting, putting more emphasis on certain elements and less emphasis on others: "It would be possible to look through all of the photographs many times and still miss what's so unusual. [...] My grandfather's right hand is never holding anything [...]" (Foer, 2003: 166). A subjective type of poiesis lies in the human imagination. Augustine's photo is black and white, yet Alex is confident that his assumptions regarding her hair and eye colour are accurate. He assumes a role as a painter, finishing off this uncompleted piece of art, yet this template of black and white tones could very easily receive a different look from somebody else. Another aspect of poiesis is the chosen reality being restricted to visual aesthetics. The smells, the sounds, the tastes and the touches are removed from the context. The eyes cannot take over these missing elements or recreate them: "He put it close to his face, like he wanted to smell it, or touch it with his eyes".

Photographs in one snapshot, within their mimetic field of competence, cannot depict how an action is going to take place. They are not capable of portraying the linearity of the past and the present's advancement within a single frame. One can distort one's perception of the image due to overshadowing fragments at will, displaying a kind of verisimilitude, a slightly altered reality. Natural decay, the yellowing of pictures, also varies reality into a shadow of its former self. The transparent, the obvious, is turned opaque, however the referent remains intact. This aging process is similar to 'memento mori', the reminder that the human flesh is evanescent and that death is irrevocable. Looking at older photos alone can awaken a sense of memento mori: they were once as I am now.

\section{Emotions through Photographs}

In Everything Is Illuminated, photos can be interpreted to function as human compasses, meaning that reproducing Augustine's image will enhance the probability of finding her. Augustine in a way resembles a modern day 'vera icon', which means that knowing what she looks like, increases the mental and bodily connection to her. Possessing a copy turns the referent (her) into a symbol of something much greater, something more abstract and less concrete, like hope, obsession or loss. Hope is found in Alex and grandfather's confidence of being prolific during their search to find Augustine although Alex's hope dies away, as seen by comparing his letters of $20^{\text {th }}$ July and $24^{\text {th }}$ December 1997 . Grandfather's hope, on the other side, mutates into a form of obsession as he wants to even borrow money from Alex and set off by himself: "I am unequivocal that I can find her. [...] Four days. Perhaps five. But it could not require more than a week. We were very near" (Foer, 2003: 216). His obsession could be considered an attempt to atone his guilt over the betrayal of Herschel's Jewish roots to the Nazis or he might be having hallucinations that Augustine and Herschel are fusions of the same person, and that by finding her, he will find him as well. The symbol of hope is, in addition, experienced in the naming of the memory box in the chapter "In Case": "[...], I could perceive that the words had been written and erased many times, written, erased, and written again". Lista did not want to let go hope that one day she would be confronted with the past and be able to pass on the voices of the oppressed and marginalized referents. Her hope definitely was not in vain, especially since Jonathan as a writer will disperse the repressed voices in his narratives of a fictionalized Trachimbrod, thus keeping them alive for future generations to come. Jonathan Safran Foer touches upon a spiritual topic based around hope, whether seeing or experiencing something justifies its existence or not: “"We could not find her, but that does not signify anything about whether you should believe in her.' 'What is the good of something that you cannot find?".

\section{Meta-photography}

\subsection{Photography's Likeness to Historiography}

In Everything Is Illuminated, photographs accompany the complete narration. New ones are but never explicitly mentioned. Only reproductions of Augustine's photo are, for example, posted to Alex for his grandfather's keeping. This indicates that the past can guide the present since they are orientating themselves via an old photo. The omnipresence of photos constructs a frame, metaphorically speaking, around the narration, meaning that a few discourses can be elucidated. To start off, I would like to mention that photographs resemble history in such a way that both can be (re)interpreted from multiple viewpoints, 
thus can be used to criticize and suspect popular beliefs of the past. This also shows the power relations of communication whether it proceeds symmetrically or complementary; in other words, if the emphasis is on sameness or differentness (Rechtien, 2014). History is most of the time written by the dominant groups and in the case of photos, the manipulation of a certain perspective from behind the lens and tampering of the image afterwards can distort how we think about the past. An example of colliding historical views might be Alex and Jonathan's opinions on the attitude of the Ukrainians to the Jews during WWII: "The Ukrainians, back then, were terrible to the Jews. They were almost as bad as the Nazis. [...] Look it up in the history books.' 'It does not say this in the history books"' (Foer, 2003: 62).

In the novel, Alex and Jonathan can be regarded as two separate stances of writing down history. They are both recording the search to find Augustine, yet whereas Jonathan opts for a highly fictionalized version, including how Trachimbrod in his imagination came to be, Alex remains more realistic with his written documentation of what happened. Over the course of Alex's letters to Jonathan, the tension between them grows bigger and bigger, wanting the other to erase or add certain — in some cases embellishing and invented - details:

"It seems to me that we are making the story even inferior. We often make ourselves appear as though we are foolish people, [...] We could give your grandfather two arms, and could make him high-fidelity.[...] We could even find Augustine, Jonathan, and you could thank her, and Grandfather and I could embrace, [...]" (Foer, 2003: 179).

This passage also emphasizes the fact that there are multiple versions of history and that it can be easily manipulated. As a kind of power relations aspect, we are only accompanied by Alex's (unreliable) narration of the search. His command of the English language is sometimes suspicious, and unlike Jonathan he was not always taking notes busily; therefore, his memories could be distorted. Jonathan's voice is only visible in his fictional story. His answers to Alex's letters are also only retold partially by Alex; thus, his voice does appear isolated.

\section{Conclusion}

The article has shown that the importance of photographs can be illustrated in their ability to act as a means of shedding light on constructs like truth, emotions, memory and space. Photos allow for more profound feelings to be revealed than words alone could achieve, e.g., grandfather's repressed memories of his true identity come to light, overpowering him ultimately. Photos indeed have their own form(s) of communication. However, many hypotheses may arise when attempting to correctly contextualize the communication without a translator, for example, whether Augustine is shown with her parents or if she was American etc. Therefore, photos can act as unreliable narrators, drawing fuzzy borders between reality and non-reality, and contorting absolute truth. Photos can be perceived either by expectations or by adding up all of the stimuli, leading to different culturally-influenced interpretations, as seen by Alex, his grandfather and Jonathan. It would be wrong to assume that photos purely and correctly 'show'; they can be 'made' and thus are capable of distorting the narration of what is right and wrong. They can operate as a projection screen for various emotions such as hope, obsession or even love. In Everything Is Illuminated, photographs also serve as a 'meta-photo', being integrated from the beginning to the end. This enables them to open up a forum of criticizing its similarity to constructed truth like in history, of analyzing the changes of spatial phenomena, framed throughout the narrative. Photographs are embedded within collective memory; this is how they are able to build a foundation for remembrance and, as a result, are capable of preventing heritage from being destroyed. They demonstrate that memory in photos exceeds that of orality. Last but not least, photos can illuminate forgotten memories, preventing dissociation due to trauma.

\section{References}

Encyclopedia of the early modern world. Availabele at: http://www.encyclopedia. com/topic/carnival.aspx\#1. (Accessed on June 1, 2018).

Foer, J. S. 2003. Everything is illuminated. London: Penguin. 
Grzega, J. 2011. Introduction to linguistics from a global perspective: An alternative approach to language and languages. München (Munich): LINCOM Europa.

Meyer, M. 2011. English and American literatures ( $4^{\text {th }}$ ed.). Tübingen: NarrFrancke Attempto Verlag $\mathrm{GmbH}+\mathrm{Co} . \mathrm{KG}$.

Rechtien, W. 2014. Kommunikationsmodell von Watzlawick. In Wirtz, M. A (ed.). Dorsch - Lexikon der Psychologie. Bern: Verlag Hans Huber.

Watzlawick, P., Beavin, J. H., \& Jackson, D. D. 1967. Pragmatics of human communication. New York: Norton.

Weseley, A. J., \& McEntarffer, R. 2010. Barron's AP psychology (4 ${ }^{\text {th }}$ ed.). New York: Barron's Educational Series, Inc. 